\title{
Custo-efetividade da produção de refeições coletivas sob o aspecto higiênico-sanitário em sistemas cook-chill e tradicional
}

\author{
Cost-effectiveness applied to hygienic-sanitary \\ aspects of collective meals production in \\ cook-chill and traditional systems
}

Vera Megumi KAWASAKI ${ }^{1}$

Denise Cavallini CYRILLO²

Flávia Mori Sarti MACHADO3

\section{RES U M O}

\section{Objetivo}

Avaliou-se a relação custo-efetividade de duas unidades de alimentação e nutrição, que adotam diferentes sistemas de produção de refeições (tradicional e cook-chill), localizadas no município de São Paulo.

\section{Métodos}

O método utilizado baseou-se na análise custo-efetividade, sendo empregados critérios de tempo e temperatura, estabelecidos na legislação como indicadores da efetividade dos sistemas de produção de refeições seguras sob o aspecto higiênico-sanitário. Os custos foram calculados pelo método de custo direto diferencial, tendo como base custo de insumos exclusivos a cada sistema.

\section{Resultados}

Os resultados demonstraram que o custo direto diferencial da unidade de alimentação e nutrição que adotava o sistema tradicional (unidade de alimentação e nutrição 1) foi de $R \$ 0,69$ por refeição servida, enquanto a unidade de alimentação e nutrição que adotava o sistema cook-chill (unidade de alimentação e nutrição 2) resultou em $R \$ 2,66$ por refeição servida. Em termos de efetividade, a unidade de alimentação e nutrição 1 apresentou grau de cumprimento de $27,9 \%$ dos critérios de tempo e temperatura, enquanto a unidade de alimentação e nutrição 2 apresentou 68,2\%. A alternativa mais custo-efetiva foi o sistema de produção tradicional, representado pela unidade de alimentação e nutrição 1, cuja razão custo-efetividade foi 2,484; enquanto a unidade de alimentação e nutrição 2 obteve razão custo-efetividade 3,907.

\footnotetext{
${ }^{1}$ Universidade de São Paulo, Programa de Pós-Graduação Interunidades em Nutrição Humana Aplicada. São Paulo, SP, Brasil.

2 Universidade de São Paulo, Faculdade de Economia, Administração e Contabilidade. São Paulo, SP, Brasil.

${ }^{3}$ Universidade de São Paulo, Escola de Artes, Ciências e Humanidades. Av. Arlindo Bettio, n.1000, Sala 302-A, Bloco Il, Ermelino Matarazzo, 03828-000, São Paulo, SP, Brasil. Correspondência para/Correspondence to: F.M.S. MACHADO.
} 


\section{Conclusão}

Verificou-se que as principais causas das transgressões em critérios de segurança de ambas as unidades de alimentação e nutrição referem-se a procedimentos que visam economia e qualidade sensorial em detrimento da segurança, apresentando pouca relação com diferenças inerentes aos sistemas de produção adotados. As etapas críticas podem ser controladas pela implantação de monitoramento de temperaturas dos alimentos, procedimento de baixo custo e alto potencial de melhoria do desempenho da efetividade na produção de refeições seguras.

Termos de indexação: análise custo-eficiência; controle de custos; higiene dos alimentos; serviços de alimentação.

\section{A B S T R A C T}

\section{Objective}

The purpose of this study was to evaluate the cost efficiency relationship from two food services from Sao Paulo city that adopt different collective meals production systems (conventional and cook-chill).

\section{Methods}

The method employed was based on cost efficiency analysis, using time and temperature criteria established in Brazilian laws as efficiency indicators for safety in hygiene and sanitary aspects of the collective meals production systems. Costs were calculated through the differential direct cost method, using as basis the costs of inputs that were exclusive to each production system.

\section{Results}

The results demonstrated that the differential direct cost from food services that adopted the conventional production system (food service unit1) was $R \$ 0.69$ per meal, while the cost of food services that adopted the cook-chill production system (food service unit 2) was $R \$ 2.66$ per meal. In terms of effectiveness, food service unit 1 achieved an accomplishment level of $27.9 \%$ in time and temperature criteria, while food service unit 2 achieved an accomplishment level of $68.2 \%$. The most cost-effective alternative was the conventional production system represented by food service unit 1, with a cost-efficiency ratio of 2.484; while food service unit 2 obtained a cost efficiency ratio of 3.907.

\section{Conclusion}

The main transgression causes in food safety criteria detected in both food services were related to procedures that seek improvements in sensory quality and economy instead of safety and there was little relationship between the inherent differences of the adopted production system. Critical stages can be controlled by monitoring food temperature, a low cost procedure with a high potential of improving efficiency performance in the production of safe meals.

Indexing terms: cost efficiency analysis; cost control; food hygiene; food services.

\section{N T R O D U Ç Ã O}

O segmento de refeições coletivas desempenha importante papel em termos de economia e saúde pública, na medida em que afeta o estado de saúde e o bem-estar da população por meio da qualidade do alimento que produz.

Restaurantes comerciais e estabelecimentos de refeições coletivas são responsáveis por significativa parcela dos surtos de doenças transmitidas por alimentos no Brasil|'. Essas doenças apresentam inúmeros agentes causais, desta- cando-se os agentes bacterianos como responsáveis pela maioria dos surtos. Diversos fatores determinam a sobrevivência ou multiplicação dos microorganismos no alimento, sendo o binômio tempo-temperatura uma combinação de fatores altamente eficaz no controle de microorganismos durante o processo produtivo'.

Os objetivos das Unidades de Alimentação e Nutrição (UAN) são elaborar e servir refeições adequadas sob o aspecto sensorial, nutricionalmente equilibradas e seguras, quanto à qualidade 
higiênico-sanitária, sem, entretanto, exceder os recursos financeiros previamente estabelecidos ${ }^{2,3}$.

O segmento de refeições coletivas manteve-se, durante longo período à margem das evoluções tecnológicas, fato evidenciado nos equipamentos, instalações e gestão de processos. O aumento da competitividade entre empresas incentivou a busca por novos processos tecnológicos de produção de refeições, sendo a centralização da produção uma das principais soluções adotadas ${ }^{4}$.

O sistema tradicional de produção de refeições coletivas é definido como o modo de produção no qual a etapa de preparo dos alimentos é efetuada imediatamente antes de servir as refeições, sendo as preparações mantidas em equipamentos conservadores de temperaturas adequadas e servidas no local da produção ${ }^{4}$.

No sistema centralizado cook-chill, as refeições são produzidas em uma unidade de alimentação e nutrição (cozinha central), transportadas a outros locais (cozinhas satélites) e servidas em refeitórios, sendo que o método de produção é baseado na preparação prévia dos itens do cardápio, porcionamento logo após a cocção, refrigeração em condições de temperatura controladas e armazenamento sob refrigeração, seguido de reaquecimento antes da distribuição e consumo. O sistema cook-chill prevê o emprego de equipamentos especificamente designados ao resfriamento rápido e ao reaquecimento adequado dos alimentos, como refrigeradores por ar insuflado, ou criogênicos e fornos de microondas, ou combinado, que permite o aquecimento homogêneo e sem ressecamento dos alimentos, por meio da circulação combinada de ar quente e de vapor ${ }^{5}$.

Embora o sistema cook-chill permita maior flexibilidade no período de preparo dos alimentos e redução do tamanho das instalações, há necessidade de maior rigor, em termos de condições de higiene, controle de temperatura e especificidade de equipamentos, especialmente em decorrência do risco apresentado pelo efeito cumulativo de possíveis contaminações durante as etapas de processamento ${ }^{4,6,7}$.
Para garantia da produção de refeições seguras é preciso reunir um conjunto de recursos físicos adequados, tais como instalações, equipamentos e utensílios, mão-de-obra capacitada e matéria-prima proveniente de fonte segura, que geram custos para o serviço de alimentação. Por outro lado, os benefícios advindos não podem ser mensurados por unidades monetárias e sim pelo alcance das metas estabelecidas, em termos de segurança para prevenção de doenças transmitidas por alimentos ${ }^{8}$.

O objetivo deste trabalho foi avaliar o custo-efetividade da produção de refeições coletivas seguras sob o aspecto higiênico-sanitário, em duas unidades de alimentação e nutrição que produzem segundo diferentes sistemas (tradicional e cook-chill), tendo como base parâmetros estabelecidos na legislação.

\section{M É T O D O S}

Foi realizada análise comparativa do desempenho de duas unidades de alimentação e nutrição, em pleno funcionamento, que adotam diferentes sistemas de produção de refeições coletivas (tradicional e cook-chill), pelo método de análise custo-efetividade, que compara os custos de implementação dos diferentes sistemas produtivos aos benefícios da redução dos riscos de ocorrência de doenças transmitidas por alimentos, resultando na produção de refeições seguras, sob o aspecto higiênico-sanitário. A análise custo-efetividade foi aplicada às duas unidades, de forma a determinar diferenças em termos de custos e grau de cumprimento de metas higiênico-sanitárias estabelecidas pela legislação.

A análise custo-efetividade é uma técnica que compara os custos de determinado projeto aos benefícios resultantes, expressos em diferentes unidades de medida, sendo os custos expressos em unidades monetárias e os benefícios em objetivos. As etapas fundamentais desta análise são: identificar os objetivos do projeto; traduzir os objetivos em dimensões operacionais ou metas; especificar as alternativas que serão avaliadas; 
determinar a estrutura de custos correspondente a cada alternativa, expressa em custo por beneficiário em determinado período de tempo; medir o grau de concretização dos objetivos propostos; comparar as alternativas entre si, utilizando as razões custo-efetividade, calculadas pela divisão dos custos monetários pelos efeitos expressos em unidades de produto ${ }^{9}$

As dimensões operacionais (ou metas), estabelecidas para avaliação da segurança higiênico-sanitária das refeições, basearam-se no cumprimento de $100 \%$ dos critérios de tempo e temperatura estabelecidos a cada etapa de produção das refeições, tendo como parâmetro a Portaria CVS-6/99, que regulamenta as condições higiênico-sanitárias de produção de refeições coletivas para o estado de São Paulo ${ }^{10}$. A escolha do emprego dos critérios de tempo e temperatura baseou-se na característica fundamental, de constituírem grandezas mensuráveis que expressam a probabilidade de eliminar riscos de dano à saúde do consumidor.

A coleta de dados de temperatura e tempo foi efetuada durante 20 dias em cada unidade de alimentação e nutrição, durante os meses de junho e julho de 2002. A mensuração das temperaturas foi efetuada utilizando-se termômetro digital com sensor de penetração modelo HT 680 A, fabricante Eletro-therm, com incerteza dos resultados variando em $0,2^{\circ} \mathrm{C}$. As temperaturas dos alimentos foram mensuradas ao final de cada etapa de produção. A técnica de mensuração baseou-se na recomendação do International Association of Milk, Food and Enviromental Sanitarians (IAMFES), que determina o centro geométrico como o ponto em que se registra a temperatura mais elevada do alimento, durante o resfriamento, e a temperatura mais baixa, durante o aquecimento. A desinfecção da haste do sensor foi feita com álcool 70\% entre as mensurações. A coleta de dados de tempo foi realizada simultaneamente ao registro das temperaturas ${ }^{10,11}$.

Foram analisados dois diferentes sistemas de produção de refeições coletivas adotados nas duas unidades de alimentação e nutrição, loca- lizadas no município de São Paulo. O primeiro estabelecimento (UAN1) pertencia a uma empresa do ramo da indústria de transformação, era administrado por autogestão e adotava o sistema de produção de refeições tradicional, com distribuição por auto-serviço. O segundo estabelecimento (UAN2) tinha administração terceirizada, pertencia a uma instituição filantrópica e adotava o sistema de produção centralizado associado ao cook-chill, sendo constituído de uma unidade central e quatro unidades satélites. As duas Unidades de Alimentação e Nutrição possuíam similaridade de condições higiênico-sanitárias (Boas Práticas de Manipulação implementadas).

A forma de avaliação do alcance dos objetivos (efetividade) de cada sistema de produção fundamentou-se em metodologia proposta por Cyrillo, que desenvolveu modelo de monitoramento baseado no cálculo de índices de obediência ou violação a critérios expressos em normas $^{12}$

A efetividade foi mensurada pelo Índice de Segurança (ISi), que representa a relação entre o número de medições de temperatura dos alimentos que atingiram a meta (NMMi) e o total de medições realizadas (NTMi), sendo expressa pela fórmula abaixo, na qual i representa o sistema (cook-chill ou tradicional): $\mathrm{ISi}=\frac{\mathrm{NMMi}}{\text { NTMi }}$

Consideraram-se, em cada etapa da produção, somente dados das temperaturas menos adequadas atingidas em cada preparação, em relação ao critério estabelecido para cada etapa, ou seja, a temperatura mais alta nas etapas de refrigeração, armazenamento sob refrigeração e transporte, e a temperatura mais baixa nas etapas de cocção, espera para distribuição e distribuição.

A mensuração dos recursos gastos pelas unidades de alimentação e nutrição foi efetuada por meio do método de custo direto diferencial, definido como a parcela componente do custo das refeições geradora de diferenciação entre os vários tipos de sistemas de produção existentes. O custo direto diferencial considera somente valores de insumos necessários à operação de um 
sistema que estejam ausentes na composição dos custos do outro sistema, ignorando custos presentes simultaneamente em ambos.

Consideraram-se como custos variáveis no cálculo do custo direto diferencial: o custo dos gêneros alimentícios referente às sobras (CALi), o custo da mão-de-obra diretamente envolvida na produção (CMOi) e o custo da energia consu-mida pelos equipamentos diferenciais de cada sistema (CENi). O custo fixo foi representado pelo custo dos equipamentos específicos de cada sistema (CEQi).

O custo das edificações não foi considerado no custo direto diferencial, porque as instalações físicas das UAN eram diferentes em vários aspectos não relacionados ao sistema de produção. O transporte dos alimentos entre a cozinha central e as satélites da UAN 2, não entrou na composição do custo direto diferencial, porque o custo seria determinado pela distância e modalidade do transporte, também não influenciando os custos de produção.

O cálculo do custo direto diferencial é representado pela fórmula abaixo, na qual i representa sistema cook-chill ou sistema tradicional:

$$
\mathrm{CDDi}=\mathrm{CALi}+\mathrm{CMOi}+\mathrm{CENi+CEQi} \text {. }
$$

As sobras representam desperdício de alimentos produzidos e não servidos. A consideração das sobras na composição do custo diferencial baseia-se no pressuposto de que os diferentes sistemas de produção de refeições apresentam diferentes taxas de desperdício, resultando em diferenças no custo de operação de cada sistema. A pesquisa de sobras das preparações foi realizada durante 16 dias em cada unidade de alimentação e nutrição. O cálculo do custo baseou-se nas sobras do arroz, do feijão e das preparações principais, cujo alimento base são as carnes ou seus derivados. Os ingredientes cárneos foram incluídos porque representavam cerca de $46 \%$ do custo de gêneros alimentícios das UAN pesquisadas, e o arroz e o feijão representavam volume significativo, em termos quantitativos, presentes diariamente nos cardápios. A apuração do custo dos alimentos referente às sobras envolveu três fases: elaboração das fichas técnicas, pesagem das sobras e cálculo do custo.

As fichas técnicas de produção e custo são receitas padronizadas das preparações que compõem um cardápio, contendo informações claras e precisas que possibilitem a reprodutibilidade da preparação ${ }^{13,14}$.

Para pesagem dos alimentos foi utilizada uma balança eletrônica modelo PRIX III, fabricante Toledo, capacidade de pesagem de 15 quilogramas e intervalos de 5 gramas.

O cálculo do custo das sobras foi efetuado com base em preços iguais para os ingredientes das preparações pesquisadas nas duas unidades de alimentação e nutrição (preços de aquisição do mês de outubro de 2002 da UAN1, complementados pelos preços de aquisição da UAN2, no caso de ingredientes presentes somente na UAN2), visando minimizar diferenças decorrentes de diferentes capacidades de compra.

O método de análise estatística utilizado para análise do Custo Direto Diferencial dos alimentos foi o teste " $t$ " de Student de diferença de médias.

O custo da mão-de-obra foi definido como a razão entre o valor total gasto com pessoal diretamente envolvido na produção de refeições e o número de refeições servidas em determinado período. As bases para determinação dos valores dos salários foram o piso da categoria, estabelecido pelo Sindicato das Empresas de Refeições Coletivas (SINDERC), e o salário de mercado, em função do cargo cotado pela Associação Brasileira das Empresas de Refeições Coletivas (ABERC) referente ao ano de $1998^{15}$.

Os equipamentos específicos ao sistema de produção cook-chill, cujos custos foram inclusos no cálculo do custo direto diferencial, foram: célula de resfriamento rápido, câmara fria de armazenamento de alimentos cozidos, equipamentos necessários ao adequado acondicionamento (carros e recipientes gastronorm), geladeiras e fornos combinados das cozinhas satélites. Fornos combinados da cozinha central da UAN2 não 
foram computados, tendo em vista que são empregados tanto no reaquecimento das preparações quanto na cocção de alimentos, similarmente às funções efetuadas pelo forno combinado presente na UAN1. Nenhum equipamento diferencial foi identificado no sistema tradicional.

O custo dos equipamentos diferenciais foi convertido em custo de serviço do capital por refeição, valor composto por: custo de oportunidade do capital gasto na aquisição do equipamento: retorno esperado caso valor idêntico fosse empregado em aplicação financeira, em vez de utilizado na aquisição de equipamento para atividade produtiva específica; custo da depreciação: razão entre o valor do investimento e a vida útil do equipamento (em dias); custo da manutenção preventiva.

O custo da energia refere-se ao consumo dos equipamentos diferenciais, ou seja, o custo da energia necessária ao funcionamento dos equipamentos existentes na UAN2 específicos do sistema cook-chill, cálculo baseado em uma estimativa do tempo de funcionamento dos equipamentos e das informações sobre o consumo de energia fornecida pelos fabricantes.

A comparação entre as duas alternativas de sistemas de produção de refeições foi realizada pelo cálculo da Relação Custo-Efetividade (RCEi), que constitui a razão entre o Custo Direto Diferencial (CDDi) por unidade de refeição servida na Unidade de Alimentação e Nutrição e o Índice de Segurança (ISi) de cada sistema: $\mathrm{RCE}=\frac{\mathrm{CDDi}}{\mathrm{ISi}}$

\section{RESULTADOS}

O número de medições de temperatura dos alimentos está apresentado na Tabela 1, dividido por etapas do processo de produção.

Na UAN1 foram pesquisadas quatro etapas (cocção, reaquecimento, espera para distribuição e distribuição), totalizando 122 medições. Vale observar que a etapa 6 é referente a produtos adquiridos prontos refrigerados, tendo sido apenas reaquecidos na UAN.

Na UAN2 o número de medições realizadas na cozinha central foi 188 e nas cozinhas satélites 667, somando 855 medições. Na cozinha central foram monitoradas as temperaturas das etapas de cocção, de refrigeração e de armazenamento sob refrigeração de todo alimento preparado, e as etapas de reaquecimento, de espera para distribuição e de distribuição dos alimentos servidos no refeitório existente na

Tabela 1. Distribuição das medições de temperatura segundo etapas de preparação em duas unidades de alimentação e nutrição (UAN1 e UAN2)(1). São Paulo, 2002.

\begin{tabular}{|c|c|c|c|c|c|c|c|c|}
\hline \multirow{3}{*}{ Etapa } & \multirow{2}{*}{\multicolumn{2}{|c|}{ UAN1 }} & \multicolumn{6}{|c|}{ UAN2 } \\
\hline & & & \multicolumn{2}{|c|}{ Central } & \multicolumn{2}{|c|}{ Satélites } & \multicolumn{2}{|c|}{ Geral } \\
\hline & $n$ & $\%$ & $\mathrm{n}$ & $\%$ & $\mathrm{n}$ & $\%$ & $n$ & $\%$ \\
\hline 1 & 30 & 24,59 & 42 & 22,34 & - & - & 42 & 4,91 \\
\hline 2 & - & - & 42 & 22,34 & - & - & 42 & 4,91 \\
\hline 3 & - & - & 9 & 4,79 & 116 & 17,39 & 125 & 14,62 \\
\hline 4 & - & - & - & - & 85 & 12,74 & 85 & 9,94 \\
\hline 5 & - & - & - & - & 124 & 18,60 & 124 & 14,50 \\
\hline 6 & 4 & 3,28 & 41 & 21,81 & 136 & 20,39 & 177 & 20,70 \\
\hline 7 & 43 & 35,25 & 7 & 3,72 & 84 & 12,59 & 91 & 10,65 \\
\hline 8 & 45 & 36,88 & 47 & 25,00 & 122 & 18,29 & 169 & 19,77 \\
\hline Total & 122 & 100,00 & 188 & 100,00 & 667 & 100,00 & 855 & 100,00 \\
\hline
\end{tabular}

1: cocção; 2: refrigeração; 3: armazenamento sob refrigeração na cozinha central; 4: transporte; 5: armazenamento sob refrigeração na cozinha satélite; 6: reaquecimento; 7: espera para distribuição; 8=distribuição; [ - ]: etapa não conduzida.

(1): Dados de junho/2002 para UAN1 e julho/2002 para UAN2. 
própria unidade central. Para as cozinhas satélites foram consideradas as temperaturas de monitoramento das etapas de armazenamento sob refrigeração na cozinha central, de transporte, de armazenamento sob refrigeração na cozinha satélite, de reaquecimento, de espera para distribuição e de distribuição.

Os índices de adequação de temperatura aos parâmetros estabelecidos, representados pelo Índice de Segurança (IS), são apresentados na Tabela 2, distribuídos segundo as etapas de produção.

Os índices de segurança foram 0,279 na UAN 1 e 0,682 na UAN2, ou seja, 27,9\% das medições de tempo e temperatura realizadas na UAN1 atenderam os critérios estabelecidos, ao passo que na UAN2, $68,2 \%$. A UAN2 teve melhor efetividade em todas as etapas de produção em relação à UAN1, assim, pode-se afirmar que ela produz refeições mais seguras do que a UAN1.

Em termos de critérios de tempo, ambas as unidades de alimentação e nutrição apresentaram $100 \%$ de obediência.

A análise do custo diferencial dos alimentos referente às sobras incluiu 18 preparações na UAN1 e 25 preparações na UAN2. Observou-se

Tabela 2. Distribuição dos índices de segurança segundo etapas de preparação em duas unidades de alimentação e nutrição (UAN1 e UAN2)(1). São Paulo, 2002.

\begin{tabular}{lcccc}
\hline \multirow{2}{*}{ Etapa } & UAN1 & \multicolumn{3}{c}{ UAN2 } \\
\cline { 3 - 5 } & & Central & Satélites & Geral \\
\hline 1 & 0,667 & 0,952 & - & 0,952 \\
2 & - & 0,810 & - & 0,810 \\
3 & - & 0,444 & 0,526 & 0,520 \\
4 & - & - & 0,929 & 0,929 \\
5 & - & - & 0,186 & 0,186 \\
6 & 0,250 & 0,976 & 0,765 & 0,814 \\
7 & 0,186 & 0,286 & 0,988 & 0,934 \\
8 & 0,111 & 0,255 & 0,828 & 0,669 \\
\hline Geral & 0,279 & 0,702 & 0,676 & 0,682 \\
\hline
\end{tabular}

1: cocção; 2: refrigeração; 3: armazenamento sob refrigeração na cozinha central; 4: transporte; 5: armazenamento sob refrigeração na cozinha satélite e 6: reaquecimento; 7: espera para distribuição; 8: distribuição; [ - ]: etapa não conduzida.

(1): Dados de junho/2002 para UAN1 e julho/2002 para UAN2. que o custo das sobras por refeição servida é maior na UAN2 do que na UAN1 (nível de significância $1 \%$ ) em decorrência da maior sobra física. Interferências no resultado de outros fatores, tais como preços dos ingredientes e tipo de preparações, foram analisadas por meio da comparação do custo por quilograma das preparações e estas não apresentaram diferença (nível de significância $5 \%)$.

O custo da mão-de-obra a valor de mercado, sem encargos, expresso por refeição servida, calculado pela razão entre a somatória dos salários do pessoal operacional e o número total de refeições servidas no mês apresentado, foi maior na UAN2. A produtividade da mão-de-obra, verificada pelo número de refeições diárias produzidas por funcionário, foi mais expressiva na UAN1 152 refeições) do que na UAN2 (27 refeições).

O custo unitário diferencial dos equipamentos (custo de serviço do capital por refeição servida) representa o valor do investimento realizado na UAN2 para viabilização do sistema cook-chill. Os equipamentos responsáveis pela manutenção da cadeia fria representaram 57,7\% dos custos dos mesmos, seguidos pelos custos destinados ao reaquecimento $(39,5 \%)$ e pelos recipientes e carros cantoneira utilizados para o armazenamento $(2,8 \%)$.

A maior parcela do custo de energia $(62,0$ $\%)$ foi representada pelo gás e pela energia elétrica que alimentavam os fornos combinados utilizados no reaquecimento das refeições. A energia elétrica gasta para manutenção da cadeia fria (célula de resfriamento, câmara fria e geladeiras destinadas ao armazenamento dos alimentos prontos) foi responsável por $38,0 \%$ do custo.

O custo direto diferencial por refeição servida, resultante da somatória dos custos dos alimentos, da mão-de-obra, dos equipamentos e da energia, foi $R \$ 0,69$ para a UAN1 e $R \$ 2,66$ para UAN2, indicando que a UAN2 apresentou custo significativamente maior do que a UAN1.

A Relação Custo-Efetividade (RCE), resultante da razão entre o Custo Direto Diferencial 
Tabela 3. Custo direto diferencial (CDD), índice de segurança (IS) e relação custo-efetividade (RCE) em duas unidades de alimentação e nutrição (UAN1 e UAN2) ${ }^{(1)}$. São Paulo, 2002.

\begin{tabular}{lll}
\hline & UAN1 & UAN2 \\
\hline CDD $^{(2)}$ & 0,693 & 2,665 \\
IS & 0,279 & 0,682 \\
RCE & 2,484 & 3,907 \\
\hline
\end{tabular}

(1) Dados de junho/2002 para UAN1 e julho/2002 para UAN2; ${ }^{(2)}$ preços de outubro/2002.

(CDD) e o Índice de Segurança (IS), foi 2,484 para UAN1 e 3,907 para UAN2, demonstrando que a UAN1 é mais custo-efetiva do que a UAN2. Em outras palavras, a UAN1 oferece um custo inferior por refeição servida para cada ponto percentual de segurança obtido (Tabela 3).

\section{I S C U S S Ã O}

A UAN1 apresentou menor efetividade na elaboração de refeições seguras em relação à UAN2, embora seja mais custo-efetiva.

As etapas de produção da UAN1 que apresentaram os menores índices de segurança foram distribuição e espera para distribuição. Os fatores determinantes foram as baixas temperaturas dos equipamentos de conservação (estufas), visando à preservação das características sensoriais, a composição do cardápio no qual predominavam preparações secas, como frituras e grelhados, que esfriam mais rapidamente que as preparações com molho, e a manutenção de sobras do almoço em temperatura inadequada para serem reaproveitadas no jantar.

Quanto ao índice de segurança da etapa de cocção, os melhores resultados também foram encontrados na UAN2. Neste caso, as características do próprio sistema favoreceram os critérios de cocção, pois se utilizava predominantemente o calor úmido para o cozimento, técnica mais adequada para elaboração de pratos que passarão por reaquecimento posterior. Preparações com caldo ou mesmo as grelhadas ou fritas, mas recobertas com molho, têm as suas características sensoriais melhor preservadas durante o reaquecimento e também contribuem para a conservação da temperatura durante as etapas de espera para distribuição e de distribuição.

A etapa mais crítica observada na UAN2 foi a de armazenamento sob refrigeração nas cozinhas satélites, indicando oscilação de temperatura na cadeia fria. A garantia de segurança no sistema cook-chill encontra-se principalmente no armazenamento a baixas temperaturas, o que permite o alongamento do prazo de validade dos produtos. Relatos na literatura indicam que surtos de doenças transmitidas por alimentos foram causados por sistemas cook-chill imperfeitos, dessa forma, qualquer falha nesse processo representa risco ${ }^{7,16}$.

Outras duas etapas com baixos índices de segurança observadas na UAN2 foram as de espera para distribuição e de distribuição da unidade central. A causa foi a mesma encontrada na UAN1, ou seja, a utilização de temperaturas inadequadas devido à preocupação com a preservação das características sensoriais e custo.

O cálculo do custo direto diferencial dos alimentos da UAN2 indicou que a utilização do sistema cook-chill não reduziu o custo por diminuição das sobras, conforme pressuposto neste estudo. Apresentou independência em relação ao tipo de sistema de produção adotado sendo, portanto, inadequado à composição do custo direto diferencial total nesta pesquisa. A eliminação do custo das sobras de alimentos na composição do custo direto diferencial total resultou em custo direto diferencial total de $\mathrm{R} \$ 0,29$ para a UAN1 e de $R \$ 1,22$ para UAN2; sendo a Relação Custo Efetividade 1,065 para UAN1 e 1,788 para UAN2, permanecendo a primeira alternativa como a mais custo-efetiva. O resultado deste estudo não invalida a possibilidade de utilização do Custo Direto Diferencial de alimentos referente às sobras, em outras pesquisas em que sejam considerados serviços de alimentação com cardápio fixo ou com refeições subseqüentes com volume significativo, utilizando o mesmo cardápio, que permita o reaproveitamento seguro dos alimentos. 
O custo da mão-de-obra na UAN2 é superior ao da UAN1, confirmando os resultados de uma extensa pesquisa realizada junto a 66 dirigentes de hospitais nos EUA ${ }^{17}$.

Variáveis, tais como o grau de complexidade dos cardápios das UAN, que poderiam consumir maior carga horária de trabalho e salários, foram analisadas e não apresentaram relação com o custo de mão-de-obra superior da UAN2. Presume-se que o principal fator associado foi a escala de operações (diferença de 14.024 refeições por mês a maior, para UAN1), porém seriam necessários outros trabalhos em que fossem comparadas unidades que apresentassem maiores semelhanças do que as pesquisadas neste estudo, para confirmar a eficiência dos sistemas em relação aos custos de mão-de-obra. Na área hospitalar, pesquisas mostram que a utilização do sistema cook-chill é mais freqüente em hospitais com 500 leitos ou mais, indicando que este sistema é adotado em unidades com maior escala ${ }^{18}$.

O custo de investimento em equipamentos no sistema cook-chill resultou em aumento da segurança das refeições, porém não resultou na economia de mão-de-obra, divergindo da literatura ${ }^{19}$.

O custo de consumo de energia para manutenção dos equipamentos referentes ao sistema cook-chill indicou gasto superior, sendo que a maior parcela de energia foi utilizada no reaquecimento dos alimentos, confirmando dados de outras pesquisas ${ }^{19,20}$.

\section{O N CLUS Ã O}

As causas das transgressões, em termos de segurança dos alimentos, na UAN1, são altamente relacionadas a procedimentos operacionais que visam economia e qualidade sensorial em detrimento da segurança, apresentando pouca relação com diferenças inerentes aos sistemas de produção adotados.

Os indicadores apontam necessidade de reavaliação dos processos produtivos adotados na
UAN1 e de intervenções nas etapas de produção mais críticas, para aumento da efetividade em segu-rança.

Tais etapas podem ser controladas pela mera implantação de monitoramento das temperaturas dos alimentos, procedimento que requer somente treinamento de funcionários e aquisição de termômetros, ou seja, uma ação que apresenta baixo custo e alto potencial de melhoria do desempenho da UAN1, em termos de efetividade na produção de refeições seguras sob o ponto de vista higiênico-sanitário.

O método cook-chill apresenta vantagens indubitáveis para sistemas de produção centralizados quanto ao aspecto segurança do alimento, tendo em vista o alto Índice de Segurança obtido pela UAN2; entretanto, devem-se considerar os riscos intrínsecos ao sistema, que exige rígido controle de tempo e temperatura nas etapas de produção.

Observou-se que a UAN2 realiza monitoramento e registro do tempo e temperatura das etapas do processo produtivo, porém, não há tabulação de dados para posterior análise, falha que ocorre sistematicamente em diversas unidades de alimentação e nutrição brasileiras, resultando em ações corretivas pontuais e ineficazes.

A utilização de um indicador, como o Índice de Segurança, é útil para a identificação das etapas mais críticas, permitindo, dessa forma, orientar a prioridade das ações corretivas e o acompanhamento dos resultados.

A aplicação do método análise custo-efetividade para determinação da melhor alternativa de produção de refeições seguras merece novos estudos, porque os serviços de alimentação são sistemas bastante complexos, envolvendo muitas variáveis a serem consideradas, e a sua aplicação exige semelhanças entre os sistemas.

\section{REFERÊ N CIAS}

1. Silva Jr EA. Manual de controle higiênico-sanitário em alimentos. 4a.ed. São Paulo: Varela; 2001. 
2. Gandra YR, Gambardella AMD. Avaliação de serviços de nutrição e alimentação. São Paulo: Sarvier; 1983.

3. Nieto RA, Vega IF, Viesti I, Kamamura H, Gambardella AMD. Modelo para análise do sistema decisório num serviço de nutrição. Rev Adm. 1986; 21(3):32-9.

4. Proença RPC. Inovação tecnológica na produção de alimentação coletiva. Florianópolis: Insular; 1997.

5. Kinton R, Ceserani $\vee$, Foshett D. Enciclopédia de serviços de alimentação. São Paulo: Varela; 1998.

6. Kaud FA. Implementing the chilled food concept. Hospitals. 1972; 46:97.

7. Lacey RW. The cook-chill crisis. Med Leg J. 1990; 88(1):18-28.

8. Kawasaki VM. Custo-efetividade da produção de refeições coletivas seguras sob o aspecto higiênico-sanitário em sistemas cook-chill e tradicional [dissertação]. São Paulo: Universidade de São Paulo; 2003.

9. Cohen E, Franco R. Evaluación de proyectos sociales. Buenos Aires: Grupo Editor Latinoamericano; 1988

10. São Paulo. Portaria CVS n.6 de 10 de março de 1999. Aprova o regulamento técnico que estabelece os parâmetros e critérios para o controle higiênico-sanitário em estabelecimento de alimentos. Diário Oficial do Estado de São Paulo. 199912 mar; Seção I: 24-27.

11. International Association of Milk, Food and Environmental Sanitarians. Guia de procedimentos para implantação do método de análise de perigos e pontos críticos de controle. São Paulo: Ponto Crítico; 1997.
12. Cyrillo DC. Reconstruindo instituições: o caso da norma brasileira para comercialização de alimentos para lactentes (NBCAL) [tese de livre docência]. São Paulo: Universidade de São Paulo; 2001.

13. Teichmann ITM. Tecnologia culinária. Caxias do Sul: EDUCS; 2000.

14. Philippi ST. Nutrição e técnica dietética. Barueri: Manole; 2003.

15. Associação Brasileira das Empresas de Refeições Coletivas [acesso em 17 fev 2003]. Disponível em: http://www.aberc.com.br

16. Blakeslee KM, Penner KP. A case study of a school foodservice cook-chil operation to develop a hazerd analysis critical control point program. Dairy Food Environ Sanit. 1999; 19(4):257-67.

17. Greatouse KR, Gregoire MB, Spears MC, Richards $V$, Nassar RF. Comparison of conventional, cookchill and fook-freeze foodservice systems. J Am Diet Assoc. 1989; 89(11):1066-611.

18. Nettles MF, Gregoire MB. Operational characteristics of hospital foodservice departments with conventional, cook-chill and cook-freeze systems. J Am Diet Assoc. 1993; 93(10):1161-63.

19. Herz ML, Souder Jr JJ. Preparation systems have significant effect on costs. Hospitals. 1979; 53(1):89-92.

20. McProud LM, David BD. Energy use and management in production of entrées in hospital food service systems. J Am Diet Assoc. 1982; 81(8):145-50

Recebido em: 20/7/2005

Versão final reapresentada em: 20/6/2006 Aprovado em: 22/12/2006 